REPRESENTATIONS OF DISCRETE FUNCTIONS 


\section{REPRESENTATIONS OF DISCRETE FUNCTIONS}

\section{EDITED BY}

Tsutomu SASAO Kyushu Institute of Technology lizuka, Japan

\section{Masahiro FUJITA \\ Fujitsu Laboratories of America Inc. Santa Clara, California, USA}

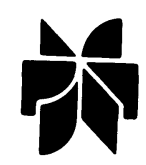

KLUWER ACADEMIC PUBLISHERS

Boston/London/Dordrecht 
Distributors for North America:

Kluwer Academic Publishers

101 Philip Drive

Assinippi Park

Norwell, Massachusetts 02061 USA

Distributors for all other countries:

Kluwer Academic Publishers Group

Distribution Centre

Post Office Box 322

3300 AH Dordrecht, THE NETHERLANDS

\section{Library of Congress Cataloging-in-Publication Data}

A C.I.P. Catalogue record for this book is available from the Library of Congress.

ISBN-13: 978-1-4612-8599-1

e-ISBN-13: 978-1-4613-1385-4

DOI: $10.1007 / 978-1-4613-1385-4$

Copyright ${ }^{\odot} 1996$ by Kluwer Academic Publishers

Softcover reprint of the hardcover 1st edition 1996

All rights reserved. No part of this publication may be reproduced, stored in a retrieval system or transmitted in any form or by any means, mechanical, photo-copying, recording, or otherwise, without the prior written permission of the publisher, Kluwer Academic Publishers, 101 Philip Drive, Assinippi Park, Norwell, Massachusetts 02061

Printed on acid-free paper. 


\section{CONTENTS}

1 GRAPH-BASED REPRESENTATIONS OF DISCRETE FUNCTIONS

Shin-ichi MINATO 1

1.1 Introduction 1

1.2 BDDs 2

1.3 Representation of Multi-Valued Functions 13

$\begin{array}{lll}1.4 & \text { Representation of Cube Sets } & 18\end{array}$

1.5 Summary 24

REFERENCES $\quad 26$

2 REPRESENTATIONS OF LOGIC FUNCTIONS USING EXOR OPERATORS

Tsutomu SASAO 29

$\begin{array}{lll}2.1 & \text { Introduction } & 29\end{array}$

2.2 Trees using EXOR Operators 30

2.3 Various AND-EXOR Expressions 36

2.4 Decision Diagrams using EXORs 43

2.5 EXOR Ternary Decision Diagrams 48

2.6 Conclusion and Comments $\quad 50$

REFERENCES 51

3 SPECTRAL TRANSFORM DECISION DIAGRAMS

Radomir S. STANKOVIĆ, Tsutomu SASAO, Claudio MORAGA 
3.1 Introduction $\quad 56$

3.2 Matrix Theory 57

3.3 BDDs and FDDs 59

$\begin{array}{lll}3.4 & \text { Generalization } & 67\end{array}$

3.5 Arithmetic Transform 68

3.6 Walsh Transform $\quad 72$

3.7 Reduced STDDs $\quad 74$

3.8 Relation Between STDDs and other DDs 77

3.9 STDDs for Arithmetic Functions 82

3.10 Conclusions and Comments $\quad 89$

REFERENCES 91

4 MULTI-TERMINAL BINARY DECISION DIAGRAMS AND HYBRID DECISION DIAGRAMS

Edmund M. CLARKE, Masahiro FUJITA, Xudong ZHAO

4.1 Introduction $\quad 93$

4.2 Multi-terminal Binary Decision Diagrams

$\begin{array}{lll}4.3 & \text { Matrix Operations } & 97\end{array}$

4.4 Spectral Transformations of Binary Valued Functions 99

4.5 Kronecker Transformations 101

4.6 Hybrid Decision Diagrams 104

4.7 Summary and Directions for Future Research 107

REFERENCES 108

\section{EDGE VALUED BINARY DECISION} DIAGRAMS

Sarma B. K. VRUDHULA, Massoud PEDRAM, Yung-Te LAI 109

$\begin{array}{lll}5.1 \text { Introduction } & 109\end{array}$

5.2 Pseudo Boolean Functions 111

5.3 Edge Valued Binary Decision Diagrams 112

5.4 The Probability Transform and its Spectrum 118

5.5 Reed-Muller Coefficients 122

5.6 Factored Edge Valued Binary Decision Diagrams 126

$\begin{array}{lll}5.7 & \text { Summary } & 130\end{array}$

REFERENCES 130 


\section{ARITHMETIC TRANSFORM OF BOOLEAN FUNCTIONS}

Jawahar JAIN

6.1 Arithmetic Transforms: Why they Need be Studied 133

6.2 An Integer-Valued Arithmetic Transform 137

6.3 More on A-Transforms: Introducing Numeric Values 145

6.4 Field Expressions and BDDs: Semi-Numeric Decision Diagrams

6.5 Application in Probabilistic Equivalence Verification 156

$\begin{array}{lll}6.6 & \text { Conclusion } & 159\end{array}$

REFERENCES 160

7 OKFDDS - ALGORITHMS, APPLICATIONS AND EXTENSIONS

Rolf DRECHSLER, Bernd BECKER

7.1 Introduction 163

7.2 Ordered Kronecker Functional Decision Diagrams 165

7.3 Basic Algorithms on OKFDDs 170

7.4 Implementation of an OKFDD Package 176

7.5 Applications and Extensions 178

$\begin{array}{lll}7.6 & \text { Conclusions } & 187\end{array}$

REFERENCES 188

8 EXACT MINIMIZATION OF FPRMS USING MULTI-TERMINAL EXOR TDDS

Tsutomu SASAO, Fumitaka IZUHARA 191

8.1 Introduction 191

8.2 Definition and Basic Properties 192

8.3 Optimization of FPRMs 195

8.4 Data Structure and Implementation 200

8.5 Optimization of Kronecker Expressions 205

8.6 Experimental Results 205

8.7 Conclusion and Comments 207

REFERENCES 209 
9 MULTIPLE DOMAIN LOGIC SYNTHESIS

Jörg BULLMANN, Udo KEBSCHULL

9.1 Introduction 211

9.2 Basics 212

9.3 The Multiple Domain Minimization Approach 221

9.4 Results 225

9.5 Conclusion 231

REFERENCES 231

10 SATISFIABILITY PROBLEMS FOR OFDDS

Ralph WERCHNER, Thilo HARICH, Rolf DRECHSLER,

Bernd BECKER

10.1 Introduction 233

10.2 Fundamental Concepts and Definitions 235

10.3 Computing Satisfying Assignments 238

10.4 Counting Satisfying Assignments 240

10.5 Conclusions 244

10.6 Proof of Theorem $2 \quad 245$

REFERENCES 247

11 COMPLEXITY THEORETICAL ASPECTS OF OFDDS

Beate BOLLIG, Martin LÖBBING, Martin SAUERHOFF,

Ingo WEGENER

11.1 Introduction 249

11.2 Improving the Variable Ordering of OFDDs is NP-complete 252

11.3 Minimal OFDD Covers 257

11.4 An Exponential Blow-Up by the Replacement of Variables by Constants 262

11.5 The Effect of Local Changes of the Variable Ordering 263

11.6 Conclusion 267

REFERENCES $\quad 267$

12 TERNARY DECISION DIAGRAMS AND THEIR APPLICATIONS

Tsutomu SASAO 
12.1 Introduction 269

12.2 Definitions and Basic Properties 270

12.3 AND TDDs 272

12.4 Reduced Ordered TDD and SOP 276

12.5 Prime TDD and Generation of Prime Implicants 278

12.6 BDDs and TDDs for Symmetric Functions 281

12.7 Experimental Results 285

12.8 Conclusion and Comments 290

REFERENCES 291

13 OR-AND-OR THREE-LEVEL NETWORKS Tsutomu SASAO 293

13.1 Introduction 293

13.2 Upper Bound on the Number of Gates 295

13.3 Lower Bound on the Number of Gates 298

13.4 Experimental Results $\quad 305$

13.5 Conclusion and Comments 307

REFERENCES 309

$\begin{array}{ll}\text { EXERCISE } & 311\end{array}$

$\begin{array}{ll}\text { APPENDIX } & 317\end{array}$

APPENDIX A $\quad 318$

APPENDIX B $\quad 324$

$\begin{array}{ll}\text { INDEX } & 327\end{array}$ 


\section{CONTRIBUTORS}

Bernd BECKER

Institute of Computer Science

Albert-Ludwigs-University

Freiburg, Germany

\section{Beate BOLLIG}

Department of Computer Science

University of Dortmund

Dortmund, Germany

\section{Jörg BULLMANN}

Wilhelm-Schickard-Institut für Informatik

Eberhard-Karls-Universität Tübingen

Tübingen, Germany

\section{Edmund M. CLARKE}

School of Computer Science

Carnegie Mellon University

Pittsburgh, PA 15213, USA

\section{Rolf DRECHSLER}

Institute of Computer Science

Albert-Ludwigs-University

Freiburg, Germany

\section{Masahiro FUJITA}

Fujitsu Labs of America Inc.

Santa Clara, CA, U.S.A.

\section{Thilo HARICH}

Computer Science Departement

J.W. Goethe-University

Frankfurt, Germany
Fumitaka IZUHARA

Department of Computer Science and Electronics

Kyushu Institute of Technology

Iizuka, Japan

\section{Jawahar J AIN}

Fujitsu Laboratories of America Santa Clara, CA, U.S.A.

\section{Udo KEBSCHULL}

Forschungszentrum Informatik

Karlsruhe, Germany

Yung-Te LAI

Hitachi Labs

179 East Tasman Dr.

San Jose, CA, U.S.A.

\section{Martin LÖBBING}

Department of Computer Science University of Dortmund

Dortmund, Germany

Shin-ichi MINATO

NTT LSI Laboratories

Kanagawa, Japan

\section{Claudio MORAGA}

Department of Computer Science Dortmund University

Germany 


\section{Massoud PEDRAM}

Department of Electrical Engineering - Systems

University of Southern California

Los Angeles, CA, U.S.A.

\section{Tsutomu SASAO}

Department of Computer Science and Electronics

Kyushu Institute of Technology

Iizuka, Japan

\section{Martin SAUERHOFF}

Department of Computer Science

University of Dortmund

Dortmund, Germany

\section{Radomir S. STANKOVIĆ}

Department of Computer Science

Faculty of Electronics

18000 Niš, Yugoslavia

\section{Sarma B. K. VRUDHULA}

Department of Electrical and Computer

Engineering

University of Arizona

Tuscon, Arizona, U.S.A.

\section{Ingo WEGENER}

Department of Computer Science

University of Dortmund

Dortmund, Germany

\section{Ralph WERCHNER}

Mathematics Departement

J.W. Goethe-University

Frankfurt, Germany

\section{Xudong ZHAO}

School of Computer Science

Carnegie Mellon University

Pittsburgh, PA 15213, U.S.A. 
In August 27-29, 1995, the IFIP WG 10.5 Workshop on Applications of the Reed-Muller Expansions in Circuit Design (Reed-Muller '95) was held in Makuhari, Chiba, Japan. Many papers focused on the representation of discrete functions. They were so instructive that we collected the most outstanding papers in a book form.

This book consists of 13 chapters. To enhance their readability, all chapters here were reviewed again and were revised. In the original papers in the proceedings, authors used their own terminology. Thus, the same notion was represented by different terminology. In the book version, however, we have insisted on a unified terminology. We include three chapters showing standard terminology. Additional examples and illustrations designed to increase the readers' understandings were also incorporated in the book version. To make the book more comprehensive, we also included two relevant chapters, which were presented at the International Workshops on Logic Synthesis. Exercises prepared by editors will make this book appropriate for a textbook of a course.

This book deals with the following topics: Binary decision diagrams (BDDs), multi-terminal binary decision diagrams (MTBDDs), edge-valued binary decision diagrams (EVBDDs), functional decision diagrams (FDDs), Kronecker decision diagrams (KDDs), binary moment diagrams (BMDs), spectral transform decision diagrams (STDDs), ternary decision diagrams (TDDs), spectral transformation of logic functions, other transformations of logic functions, EXORbased two-level expressions, FPRM minimization with TDDs and MTBDDs, complexity theories on FDDs, multi-level logic synthesis, and complexity of three-level logic networks.

In this book, a discrete function denotes a mapping $S \rightarrow L$, where $S$ and $L$ are finite non-empty sets. A switching function denotes a discrete function, where $S=\{0,1\}^{n}$ and $L=\{0,1\}$. 


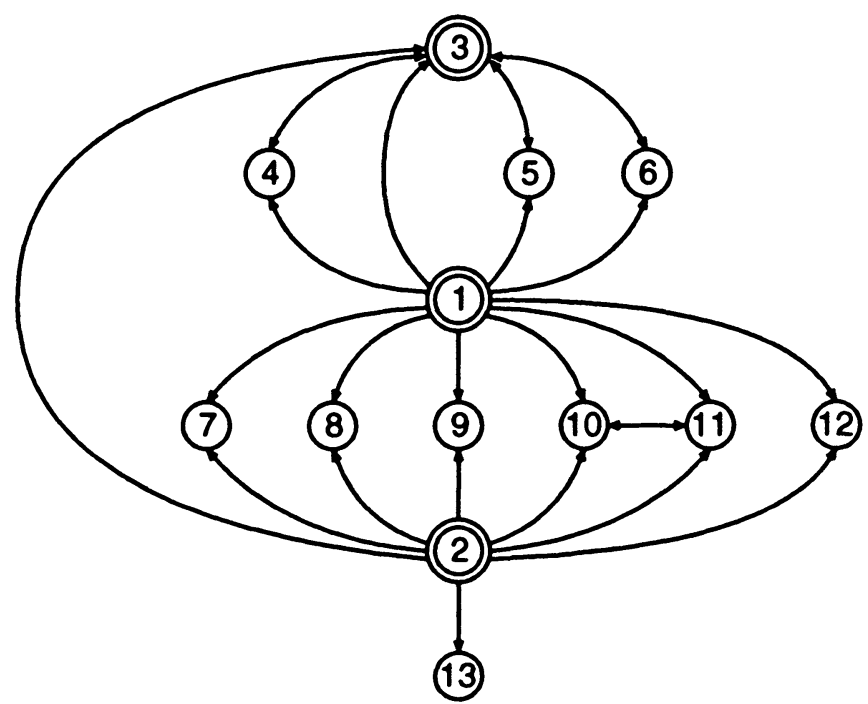

Figure 1 Relation of chapters.

\section{Overview of the Book}

This book consists of 13 chapters. Fig. 1 shows the relation of these chapters. The first three chapters are introductions. They contain many illustrations to clarify concepts presented in the text. It is recommended to read these three chapter first. After this, the other chapters will be easy to read.

Chapter 1 introduces BDDs, and their variants: it surveys the techniques for BDDs, and shows the relations between different types of decision diagrams.

Chapter 2 introduces EXOR-based representations: it compares complexities of various representations.

Chapter 3 shows relation of various decision diagrams: One decision diagram represents a function $f$, and at the same time, the spectrum $S_{f}$. For example, a BDD represents $f$, and at the same time, the Reed-Muller spectrum of $f$. It also shows methods to represent arithmetic functions by using decision diagrams. This chapter is related to Chapters 4,5 , and 6 . 
Chapter 4 shows applications of MTBDDs. It shows an MTBDD-based computation of spectral transformations, which is a revolutionary idea. With this technique, we can compute the spectra of a logic function with more than 200 variables. This chapter also proposes HDDs (hybrid decision diagrams).

Chapter 5 introduces EVBDDs, which are useful to represent both Boolean and arithmetic operations. This chapter shows EVBDD algorithms for binary operations. An extension of EVBDDs is also described.

Chapter 6 presents arithmetic transformations, which are useful for verification of large networks.

Chapter 7 considers OKFDDs (KDDs). OKFDDs are generalization of BDDs and FDDs, and can represent logic functions more compactly than BDDs and FDDs.

Chapter 8 shows a method for exact minimization of FPRMs. With this method, we could obtain an exact minimum FPRM with 94 inputs. MTBDDs, and MTTDDs are the key data structure in this method.

Chapter 9 shows a multi-level logic synthesis method. It considers both ANDOR and EXOR-based designs.

The next two chapters deal with complexity issues of FDDs.

Chapter 10 considers the satisfiability problems. It shows that the counting the satisfying assignments in an OFDD cannot be solved in polynomial time unless $N P=P$.

Chapter 11 considers the complexity of changing the order of input variables. It also shows that the replacement of variables with constants can cause an exponential blow up of the FDD size.

Chapter 12 presents ternary decision diagrams (TDDs). A TDD represents a logic expression rather than a logical function. A BDD is a special case of a TDD, where only two edges representing $f_{0}$ and $f_{1}$ are used. There is a $2 n$ variable function whose size of the TDD is $2 n^{2}+2$, while the size of the BDD is $2^{n+1}$. TDD can be a mathematical model for analyzing the complexity of Boolean functions. 
Chapter 13 deals the complexity of OR-AND-OR three-level logic networks. Any logic functions with $n=2 r$ variables are represented with at most $2^{r+1}$ gates.

Unlike the other chapters, preliminary versions of last two chapters were presented at the International Workshop on Logic Synthesis in May 1993, and 1989 , respectively.

\section{Acknowledgments}

We would like to express our appreciation to all the contributors for their participation and cooperation in this project. They patiently revised the manuscript many times to improve the readability. Many people spent their precious time for review under extremely tight time schedule: Their names appear at the end of this book. We also thank all the attendee for the IFIP WG 10.5 Workshop on Applications of the Reed-Muller Expansions in Circuit Design. Conversation at the workshop inspired improvements in the enclosed chapters. We acknowledge with gratitude the support from the Fujitsu Systems Laboratory, The Telecommunication Advancement Foundation (TAF), and the Chiba Convention Bureau. Prof. Kazuhiko Iwasaki did a very good job as the local arrangement chairman.

Students of Sasao's Laboratory made important suggestions to improve the readability of the chapters during seminars using the preliminary version of the book.

Finally, we would like to thank Mr. Debatosh Debnath and Mr. Munehiro Matsuura for editing the $\mathrm{LT}_{\mathrm{E}} \mathrm{X}$ files to produce the camera-ready copy. Without their help, the book would never have materialized.

Tsutomu Sasao, Iizuka, Japan.

Masahiro Fujita, Santa Clara, California, U.S.A. 